\title{
Photoinduced Electron Transfer in Donor-Acceptor Complexes: Isotope Effect and Dynamic Symmetry Breaking
}

\author{
Jan Paul Menzel, Huub J. M. de Groot, ${ }^{\circledR}$ and Francesco Buda*(† \\ Leiden Institute of Chemistry, Leiden University, PO Box 9502, 2300 RA Leiden, The Netherlands
}

Supporting Information

ABSTRACT: Electron-nuclear (vibronic) coupling has emerged as an important factor in determining the efficiency of energy transfer and charge separation in natural and artificial photosynthetic systems. Here we investigate the photoinduced chargetransfer process in a hydrogen-bonded donor-acceptor molecular complex. By using real-time quantum-classical simulations based on time-dependent Kohn-Sham equations, we follow in detail the relaxation from the Franck-Condon point to the region of strong nonadiabatic coupling where electron transfer occurs. We elucidate how the charge transfer is coupled to specific vibrational modes and how it is affected by isotope substitution. The importance of resonance in nuclear and electron dynamics and the role of dynamic symmetry breaking are emphasized. Using the dipole moment as a descriptive parameter, exchange of angular momentum between nuclear and electronic subsystems in an electron-nuclear resonant process is inferred. The performed simulations support a nonadiabatic conversion via adiabatic passage process that was

recently put forward. These results are relevant in deriving rational design principles for solar-to-fuel conversion devices.

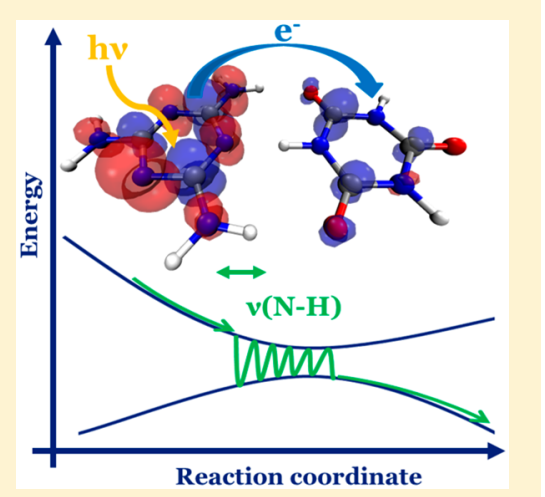

$\mathrm{P}$ hotoinduced charge separation is a key process in photosynthesis. In nature, extended antenna complexes collect solar energy in the form of electronic excitations, which are then transferred to a reaction center, where the actual charge separation takes place. ${ }^{1-3}$ Only after this separation can the photoenergy be converted into chemical energy. Because ultrafast charge separation in combination with spatial separation plays a major role in preventing charge recombination, understanding the origin of these fast and efficient processes is of crucial importance for the design of artificial photosynthesis devices. $^{4-8}$ Coherent charge transfer is an emerging concept, where, through vibronic coupling, nuclear vibrations resonate with specific electronic transitions, thus driving charge transfer efficiently. ${ }^{9-14}$ Experimental observations and theoretical investigations underline the role of this effect in natural ${ }^{12,15-21}$ as well as artificial systems. ${ }^{22-27}$ For example, Falke et al. identified the $\mathrm{C}=\mathrm{C}$ stretch and a pentagonal pinch mode to drive charge transfer from a polymer toward a fullerene in a polymer blend. ${ }^{25}$ However, some aspects of coherent charge transfer are not wellunderstood. In this work, using quantum-classical nonadiabatic Ehrenfest dynamics simulations, we explore which and how specific nuclear modes are selected in a donoracceptor molecular complex and what role isotope effects and dynamic symmetry breaking play. Computer simulations of these processes provide the possibility of freezing specific nuclear coordinates or bond distances to access how crucial these degrees of freedom are for the charge transfer. These exercises, though unphysical, can provide insight hardly accessible through experimental investigations. For this in silico investigation, we consider a DNA base pair mimic consisting of melamine and isocyanuric acid, ${ }^{22}$ which self- assemble through an extended two-dimensional hydrogen bonding network. ${ }^{28}$ This explicit donor-acceptor molecular complex combines relative simplicity with a realistic distribution of different chemical entities common in biological systems. ${ }^{29}$ We investigate photoinduced coherent charge transfer and follow the onset of electron transfer (ET) upon photoexcitation in real time. Through a comparison of the Fourier analysis of the nuclear and electronic motion along the same trajectory, relevant frequencies can be extracted. We find that modulating the frequency of key vibrational modes by isotope substitution changes their participation in the coherent process and increases the importance of other modes. Additionally, our investigations stress the importance of dynamic symmetry breaking for coherent charge transfer. Analogies are found between the simulation results of the photoinduced coherent charge transfer and the wellestablished adiabatic passage processes observed during NMR adiabatic pulse ${ }^{30}$ and chirped laser pulse optical spectroscopy experiments, ${ }^{31}$ providing additional support for the nonadiabatic conversion via adiabatic passage (NCAP). ${ }^{9,32,33}$ These results provide important principles to consider while designing and optimizing charge-transfer and charge-separation devices. ${ }^{12,33}$

Photoinduced Electron Transfer and Vibronic Coupling. The structure of the DNA base pair mimic (melamine and isocyanuric acid) is given in Scheme la. We fix the nuclear coordinates of the nitrogen in the melamine tail as well as the

Received: August 16, 2019

Accepted: October 8, 2019

Published: October 8, 2019 
Scheme 1. (a) Chemical Structure of the Pseudo Base Pair Melamine (left, donor) and Isocyanuric Acid (right, acceptor) and (b) Kohn-Sham Orbitals Relevant for the Photoinduced Charge Transfer ${ }^{a}$

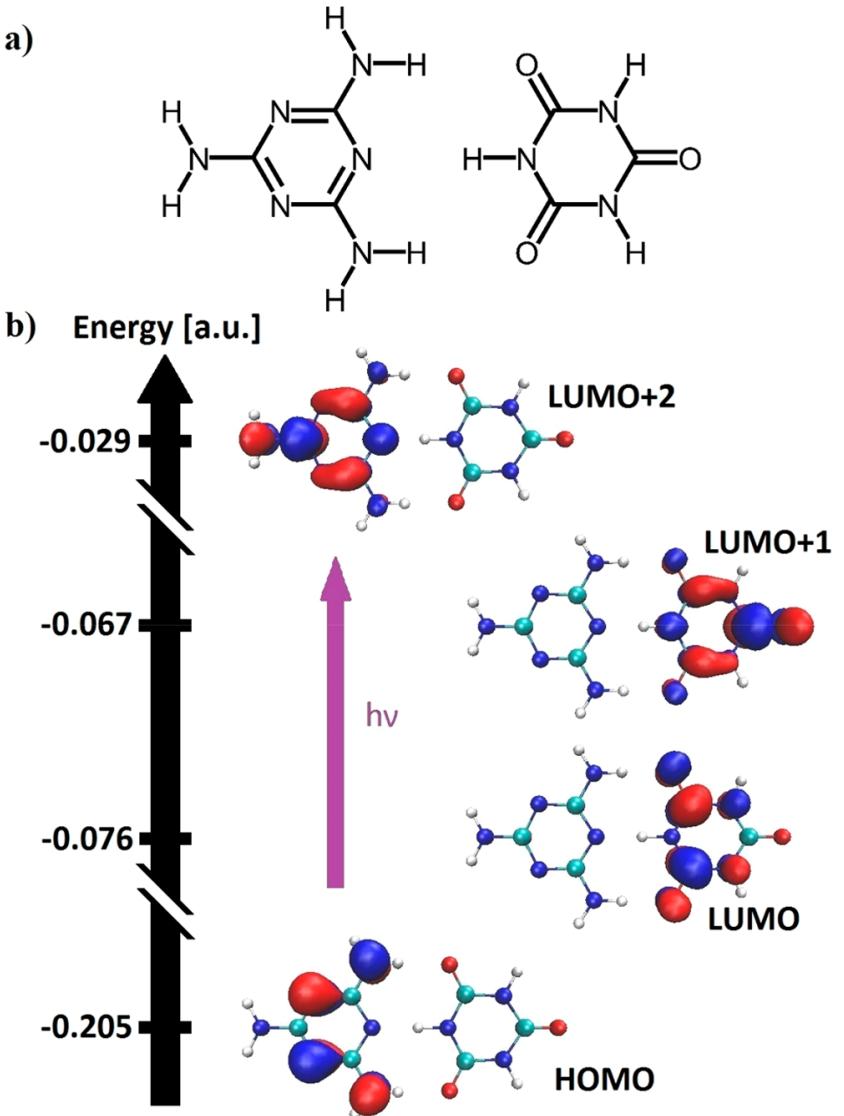

${ }^{a}$ The melamine is excited from its HOMO (HOMO of the total system) to its LUMO (the LUMO+2 of the total system), with the LUMO and LUMO+1 localized on the acceptor.

oxygen in the isocyanuric tail (furthest from the hydrogen bond interface) to avoid translational motion of the complex and to maintain the relative distance, which greatly affects the ET rate (see Figure S1 in the Supporting Information). This constraint mimics the effect of the environment in a hydrogenbonded crystal or DNA backbone. Scheme $1 \mathrm{~b}$ shows the relevant molecular orbitals involved in the photoinduced ET process. These were obtained for an optimized geometry at the DFT/BLYP ${ }^{34,35}$ level (see Supporting Information SI.1 for more computational details). Upon excitation, an electron is transferred from the melamine highest occupied molecular orbital (HOMO) to its lowest unoccupied molecular orbital (LUMO) (the LUMO+2 of the total complex, see Scheme 1b). This excitation localized on the donor is higher in energy than the charge-transfer state from HOMO to LUMO and from $\mathrm{HOMO}$ to LUMO+1. Time-dependent density functional theory (TDDFT) (both with BLYP and CAM-B3LYP) calculations have shown that the most relevant excitonic excitation has the highest oscillator strength in the energy range explored, while the charge-transfer state has a lower energy $^{22}$ (see Supporting Information SI.2), resulting in an energy gradient upon excitation from the excitonic to the charge-transfer state. Ehrenfest dynamics simulations with BLYP $^{34,35}$ as exchange-correlation functional are performed using the octopus program, ${ }^{36-39}$ starting from this excitonic state localized on the donor by changing the orbital occupations: the $\beta$-HOMO occupation was changed to 0 , while the $\beta$-LUMO+2 occupation was adapted to 1 , representing a local excitation on the melamine as shown in Scheme $1 \mathrm{~b}$. A time step of 1 attosecond is used for a total simulation length of 100 fs (for more details of the Ehrenfest dynamics see Supporting Information SI.1). Upon instantaneous excitation in the Franck-Condon region, the system starts to relax toward a more preferable geometry.

To quantify the electron transfer along the trajectory, we integrate the excess $\beta$-spin density localized on the acceptor (see Supporting Information SI.1 for detailed information). Because one $\beta$-electron is moved from the HOMO to the $\mathrm{LUMO}+2, \beta$-spin density represents the density of the excited electron, while a lack of $\beta$-spin can be associated with the hole. The integrated (excited) electron density on the acceptor is reported over the trajectory from 0 to 100 fs in Figure $1 \mathrm{a}$.

An oscillating character of the ET process, starting at about $10 \mathrm{fs}$, is clearly visible. The inset (top of Figure 1) shows the difference spin density at the beginning and end of the simulation; at the beginning, $\mathrm{HOMO}$ and $\mathrm{LUMO}+2$ can clearly be seen in the respective hole and electron densities. At the end of the simulation, electron density has been transferred to the acceptor molecule partially populating the $\mathrm{LUMO}+1$ and LUMO. The time evolution of the orbital energies is shown in Figure 1d. After initial photoexcitation into the $\mathrm{LUMO}+2$, a quick relaxation away from the Franck-Condon region takes place, bringing the orbital energy of the $\mathrm{LUMO}+2$ closer to the LUMO and LUMO+1 energies as a response to the nuclear motion. The orbital energies start rapidly approaching each other, crossing at approximately $17 \mathrm{fs}$, which corresponds to the first electron-transfer maximum (see Figure 1a). After the first crossing the orbital energies keep oscillating and crossing over time. However, we should keep in mind that this long-term behavior might be due to the mean field approach used, preventing the system from collapsing to the lower potential energy surface. This is also the most likely reason why we do not observe complete conversion into the charge-transfer state. The complete time evolution of electron and hole density as well as the nuclear motion is shown in Movie S1 in the Supporting Information. Noticeably, if the nuclear coordinates are fixed in the initial optimized geometry, no electron transfer is observed showing the crucial role of nuclear dynamics (see Figure S2 in section SI.3 in the Supporting Information). The Fourier transform of the electron-transfer time evolution can provide information on the characteristic frequencies associated to this process. Similarly, the total vibrational density of states (VDOS) can be extracted from the nuclear trajectory by performing a Fourier transform of the velocity autocorrelation function. In Figure 1e, ET frequencies and the VDOS computed on the same trajectory corresponding to Figure 1a are compared. In the frequency spectrum of the ET process, four major peaks can be distinguished at 2495, 3505, 4230, and $5525 \mathrm{~cm}^{-1}$. The two higher-frequency peaks can be associated with electronic coherences. In particular, the peak at $4230 \mathrm{~cm}^{-1}$ can be assigned to electronic resonances close to the Franck-Condon point because it is prevalent in a purely electronic dynamics trajectory with fixed nuclear positions at the initial geometry (see Figure S3). A striking overlap between the VDOS and ET frequencies appears around $3500 \mathrm{~cm}^{-1}$, corresponding to an oscillation time of $9.5 \mathrm{fs}$, suggesting strong electron-nuclear 

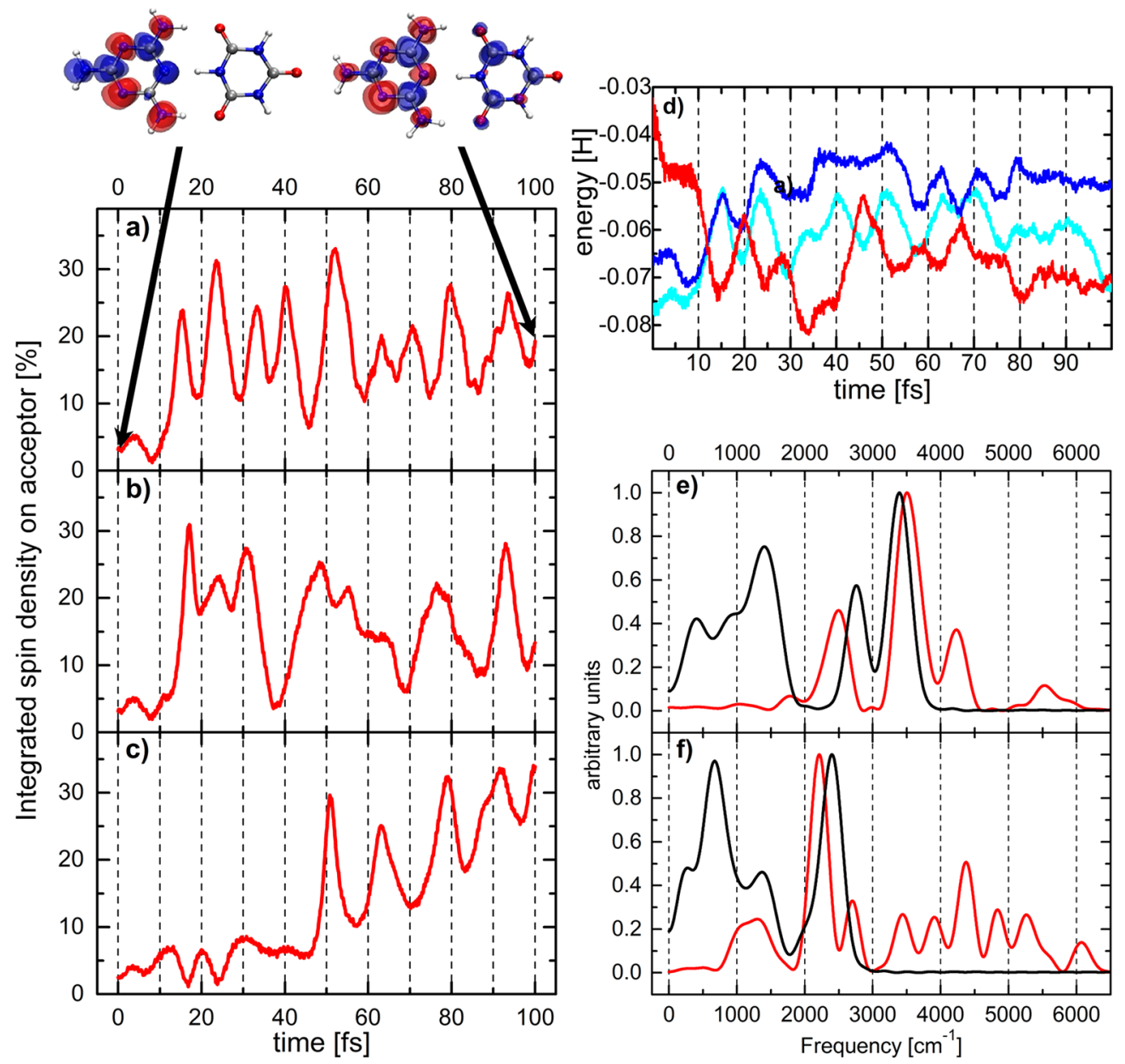

Figure 1. (a) Electron transfer from the melamine (donor) to the isocyanuric acid (acceptor) quantified by $\beta$-spin density localized on the acceptor. Shown on top are snapshots of the difference between $\alpha$ and $\beta$ spin density $(\rho(\beta)-\rho(\alpha))$ at the beginning and end of the simulation: blue shows excess $\beta$-spin density, corresponding to the excited electron, while red is the lack of $\beta$-spin density, which can be associated with the hole density. (b) Electron transfer in fully deuterated melamine-isocyanuric acid system. (c) Electron transfer for fully symmetrical starting geometry. (d) Time evolution of the orbital energies of the LUMO+2 (red), LUMO+1 (dark blue), and LUMO (light blue) during the Ehrenfest dynamics simulation corresponding to panel a. (e) Frequency spectrum associated to the electron transfer (red line) obtained by Fourier transform of the electron transfer time evolution shown in panel a and the total vibrational density of states (VDOS) of the pseudo base pair (black line) extracted from the corresponding nuclear trajectory. (f) Electron-transfer frequencies (red line) and total nuclear VDOS (black line) for the fully deuterated system corresponding to panel $b$.

coupling. This peak in the VDOS corresponds to the highestfrequency modes, the $\mathrm{N}-\mathrm{H}$ stretching vibrations (see Figures S4-S6, where peaks in the VDOS are assigned to specific nuclear motion). The lowest-frequency peak in the ET spectrum at around $2500 \mathrm{~cm}^{-1}$ shows significant overlap with a peak in the VDOS associated with the central bridging $\mathrm{N}-\mathrm{H}$ bond of the isocyanuric acid (see Figure S4). C=O stretches and other vibrational modes (below $2000 \mathrm{~cm}^{-1}$ ) have a negligible effect on the ET frequency spectrum. Therefore, the $\mathrm{N}-\mathrm{H}$ stretching modes appear to provide the important vibronic coupling, enabling charge transfer to take place.

Isotope Effect. If the $\mathrm{N}-\mathrm{H}$ bond stretches are coupled to the photoinduced coherent charge transfer in this system, changing the corresponding vibrational frequency should affect the electron-transfer process. Schnedermann et al. recently found that isotope effects play a significant role in the vibronically coherent process of photoisomerization of the 11-cis retinal. ${ }^{40}$ To explore this hypothesis, we mimic in silico an isotope substitution experiment by exchanging all hydrogen atoms by the heavier deuterium isotope. Starting from otherwise identical initial conditions, we perform an Ehrenfest dynamics simulation for the fully deuterated system.

The isotope substitution results in a modified electrontransfer pattern as shown in Figure 1b. In particular, several oscillations at various frequencies contribute to the pattern. These frequencies are shown together with the total VDOS of the corresponding nuclear trajectory in Figure 1f. The change in frequency of the $\mathrm{N}-\mathrm{D}$ stretching modes compared to the $\mathrm{N}-\mathrm{H}$ stretches is clearly visible and is proportional to about $\frac{1}{\sqrt{2}}$, as expected for a localized mode with substitution of ${ }^{1} \mathrm{H}$ by ${ }^{2} \mathrm{D}$ (see also Figure $\mathrm{S} 7$ where a direct comparison of the VDOS for the deuterated and hydrogenated systems is shown). The two distinct $\mathrm{N}-\mathrm{H}$ peaks (see Figure 1e) merge in the $\mathrm{N}-\mathrm{D}$ case, because the energy difference scales according to the isotope shift as well. The lower $\mathrm{N}-\mathrm{D}$ peak is still visible as a shoulder at around $2000 \mathrm{~cm}^{-1}$. The lower-frequency bands involve $\mathrm{C}=\mathrm{O}$ and $\mathrm{C}-\mathrm{N}$ stretches as well as ring modes and bending modes whose frequencies are not substantially affected by the isotope substitution. 
In the high-frequency region $\left(>3000 \mathrm{~cm}^{-1}\right)$, well-resolved electronic frequencies are visible in the ET spectrum. The most dominant peak in the ET frequency spectrum $\left(\sim 2500 \mathrm{~cm}^{-1}\right)$ is still resonant with the $\mathrm{N}-\mathrm{D}$ stretching, as it is red-shifted consistently with the shift in the N-D stretching frequency relative to the $\mathrm{N}-\mathrm{H}$ mode. In addition to the $\mathrm{N}-\mathrm{D}$ there is an overlap between the nuclear and electronic spectra in the lower frequency region (around $1300 \mathrm{~cm}^{-1}$ ) in contrast to the ${ }^{1} \mathrm{H}$ case. This indicates that also lower-frequency nuclear vibrations (e.g., $\mathrm{C}=\mathrm{O}$ and $\mathrm{C}-\mathrm{N}$ stretching) couple with the ET process. These effects show that the resonance condition between the electronic energy difference and the nuclear vibrational frequencies changes upon isotope substitution. From this result we can already conclude that the $\mathrm{N}-\mathrm{H}$ stretching is not uniquely essential for facilitating the electron transfer. What is important, is that these $\mathrm{N}-\mathrm{H}$ modes, because of their high frequencies, are the first nuclear vibrations to match the energy difference between the electronic states during the relaxation process. The isotope substitution moves the resonance condition to lower frequencies. This is shown in a schematic potential energy plot along a generic nuclear relaxation coordinate (Scheme 2 ). In the ${ }^{2} \mathrm{D}$ case, additional nuclear modes couple to the electronic motion in contrast to the ${ }^{1} \mathrm{H}$ case, where the $\mathrm{N}-\mathrm{H}$ frequencies are energetically isolated from all other modes.

Dynamic Symmetry Breaking. To investigate the effect of symmetry on the photoinduced charge-separation process, an Ehrenfest dynamics simulation was performed starting from an optimized geometry with enforced $C_{2 v}$ symmetry. The electron-transfer pattern during this simulation can be seen

\section{Scheme 2. Schematic Representation of the Coherent} Process in the Investigated System ${ }^{a}$

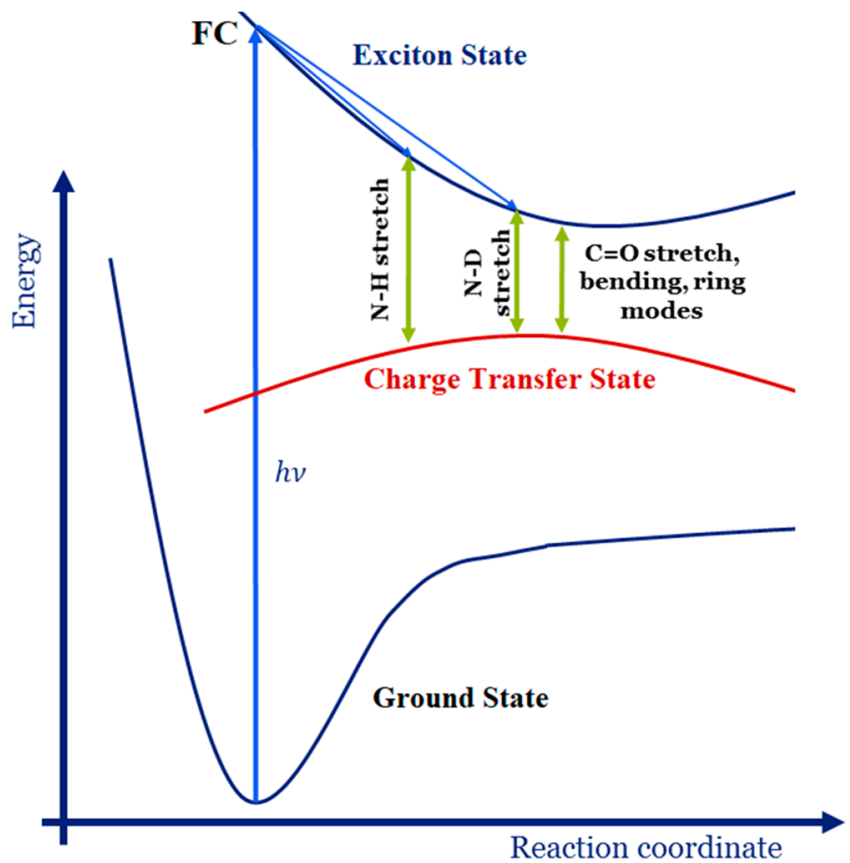

${ }^{a}$ Upon excitation, the system relaxes from the Franck-Condon point (FC). In the ${ }^{1} \mathrm{H}$ system, the first available high-energy vibrations are due to the $\mathrm{N}-\mathrm{H}$ stretching and are well-separated from the other modes. In the deuterated case, the $\mathrm{N}-\mathrm{D}$ stretching has a vibrational energy similar to several other modes, which can therefore also couple to the electronic motion. in Figure 1c, showing a delay of the first significant peak compared to the other two simulations (Figure 1a,b). This is surprising considering that the orbital energy differences are small enough for resonant coupling with available nuclear modes within the first 10 fs (see Figure S8). To explain this suppression of coherent charge transfer, we need a more detailed investigation of the initial geometric relaxation. In Figure $2 \mathrm{a}$, the time evolution of the interfacial $\mathrm{N}-\mathrm{H}$ bond distances (which were the most relevant in the previous cases) is compared with the electron transfer (Figure $2 b$ ).

Two main messages can be extracted from Figure 2a: (i) Upon electronic excitation, symmetry-equivalent bonds with respect to the $C_{2}$-axis ( $2 a$ and $2 b ; 3 a$ and $\left.3 b\right)$ are evolving in a perfectly identical manner, maintaining the $C_{2 v}$-symmetry. Only when the electron transfer starts does the deviation from $C_{2 v}$ symmetry gradually increase from around $50 \mathrm{fs}$. (ii) The amplitude of the $\mathrm{N}-\mathrm{H}$ bond oscillations increases dramatically during the electron-transfer process, suggesting that electronic energy is transferred into these nuclear vibrations.

The question arises whether the electron transfer induces this divergence from the $C_{2 v}$ symmetric motion, or conversely, it is the breaking of symmetry that allows for the electron transfer in the first place. To address this question, it is helpful to look at the difference in bond length between symmetryequivalent bonds with respect to the $\mathrm{C}_{2}$-axis. We find that the pairs of bonds that first diverge from perfect symmetric motion are $\mathrm{C}-\mathrm{N}$ bonds in the aromatic ring of the donor molecule (see Figure S9 showing all symmetry-equivalent pairs). In Figure $2 c$, the bond length difference between the two bonds marked in blue/red in the molecular structure, named 1a and $1 b / 2 a$ and $2 b$ (bond distance of $a-$ bond distance of $b$ ) are shown. After about $35 \mathrm{fs}$, the bond lengths start to diverge: bond $1 \mathrm{a}$ shortens, while $1 \mathrm{~b}$ elongates and at the same time bond $2 \mathrm{a}$ increases while bond $2 \mathrm{~b}$ decreases in length. The combination of these concerted expansions and compressions corresponds to a normal mode of the donor molecule of the $\mathrm{A}_{2}{ }^{\prime}$ irreducible representation of the $D_{3 h}$ group, which is the point group of both melamine and isocyanuric acid when in isolation. This $\mathrm{A}_{2}{ }^{\prime}$ irreducible representation also includes rotation around the $z$ axis which is oriented perpendicular to the molecular plane. This motion breaks the initial $C_{2 v}$ symmetry. Two equivalent modes rotating in opposite directions around the $z$-axis exist that could be excited. Once a small preference, for instance one due to numerical noise in the integration of the equations of motion, is given to a rotation in one direction in favor of its counter-rotating equivalent, asymmetric motion will emerge. About 45 fs into the simulation, which is around $10 \mathrm{fs}$ after the onset of the asymmetric motion, the electron-transfer process starts, responding to the breaking of symmetry. This underlines the importance of dynamic symmetry breaking in photoinduced coherent charge transfer. In a system interacting with its environment, there will always be a slight preference to one component over the other, resulting in symmetry breaking. This holds especially true when coupled to a thermal bath or in a chiral environment. The displacement from symmetric geometry at $45 \mathrm{fs}$, when the ET process starts, is about 0.01 $\AA$. This low displacement will already be present at extremely low temperatures. Still, these results already suggest a design principle for systems to optimize coherent charge transfer: breaking the symmetry as initial condition, e.g. by using chiral components. This principle holds for typical natural photosynthetic systems. ${ }^{41,42}$ 


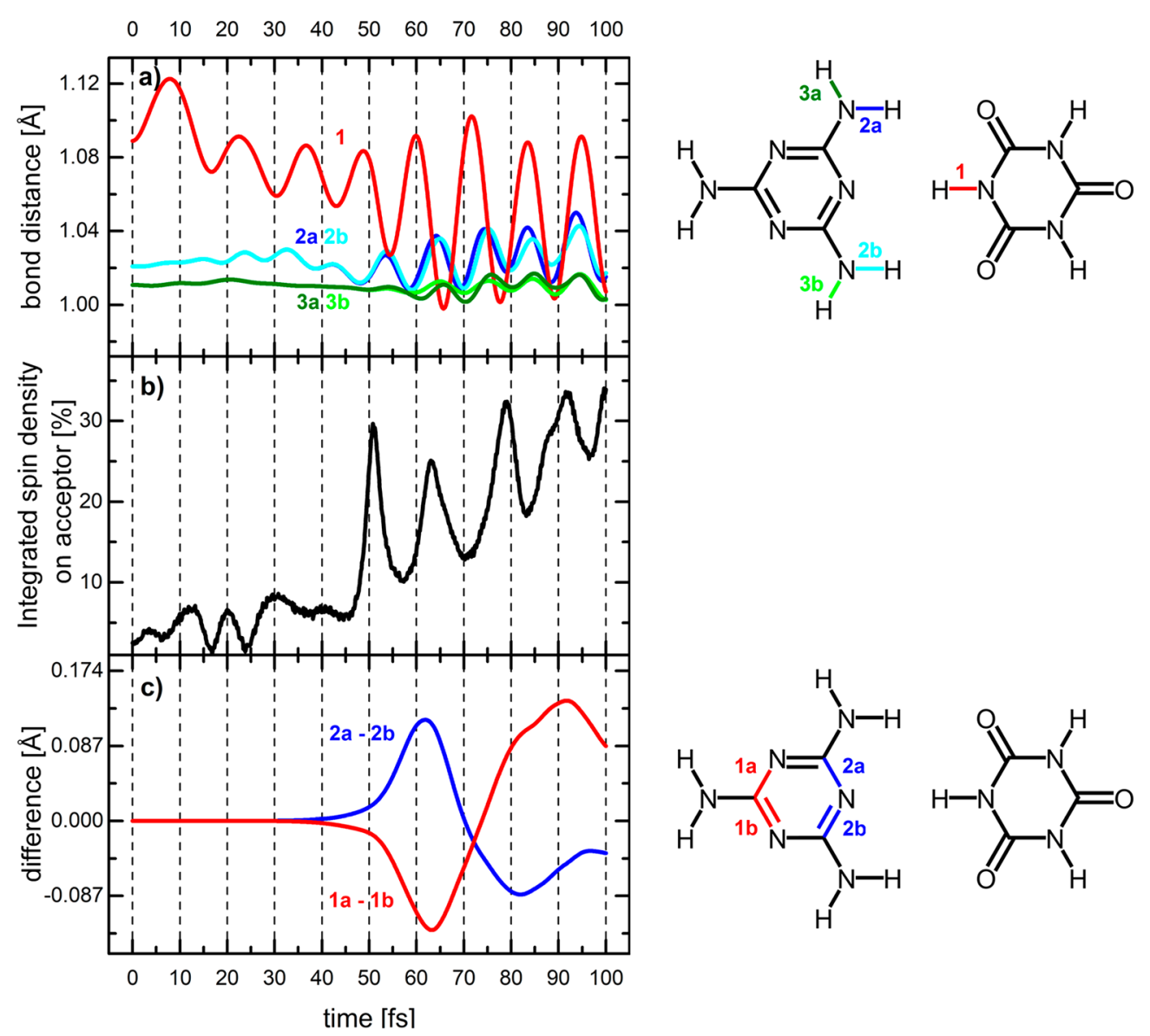

Figure 2. (a) Time evolution of the $\mathrm{N}-\mathrm{H}$ bond distances for the donor-acceptor system with enforced starting $C_{2 v}$ symmetry. Different colors correspond to different bonds indicated in the chemical structure on the right. (b) Electron-transfer pattern for the same trajectory. (c) Difference in bond length between symmetry-equivalent $\mathrm{C}-\mathrm{N}$ bonds shown in the chemical structure on the right.

Similarities with Other Adiabatic Passage Processes. We stress the similarity between the coherent charge transfer and the well-established adiabatic passage processes observed during adiabatic pulses in both NMR and optical spectroscopy: ${ }^{30,31}$ In this work we have a process that can be described as population change between two states, an excitonic and a charge-transfer state. The two states have an energy difference corresponding to a frequency $\omega_{\mathrm{e}}$ that is modulated over time because of relaxation from the Franck-Condon point (see Scheme 2). This closely mirrors the radiofrequency $\omega_{\text {rf }}$ in an NMR adiabatic pulse experiment and the laser frequency $\omega_{\mathrm{L}}$ in a chirped laser pulse in optical spectroscopy. In all three cases, we sweep toward a resonance condition, in our case $\omega_{\mathrm{e}}=\omega_{\mathrm{n}}$, with $\omega_{\mathrm{n}}$ being an available nuclear frequency. As we approach the resonance, nonadiabatic coupling increases significantly. The electronic motion slows down, and the time scales of nuclear and electronic motion converge, making exchange between the nuclear and electronic system possible (vibronic coupling). As in the two adiabatic passage processes mentioned earlier (NMR adiabatic pulse, reversal of magnetization; chirped laser pulses, change of orbital angular momentum), a change of population from the excitonic state to the charge-transfer state can be observed (see electron transfer in Figure $1 \mathrm{a}-\mathrm{c}$ ), showing characteristic oscillations of frequency $\omega_{n}$. Moving into the interaction frame corresponding to this $\omega_{\mathrm{n}}$ this exchange should then be smooth, again similar to the mentioned other processes. Within the interaction frame of the electronic frequency $\omega_{\mathrm{e}}$, however, similarly to the case of the interaction frame of the laser pulse in optical spectroscopy or the radio frequency in an adiabatic pulse experiment in NMR, there is a precession around the interaction frame axis because of the mismatch of $\omega_{\mathrm{e}}$ and $\omega_{\mathrm{n}}$ when sweeping $\omega_{\mathrm{e}}$. When the resonance condition $\omega_{\mathrm{e}}=\omega_{\mathrm{n}}$ is exactly met, the nonadiabatic coupling is maximal (see also SI.7 for an estimate) and the populations of states 1 and 2 will be exactly $1 / 2$ each. As the system moves out of resonance, with $\omega_{\mathrm{e}}$ now being smaller than $\omega_{\mathrm{n}}$, the system further evolves into full conversion (state 2 population equals 1 , state 1 population equals zero). In our simulation, because of the mean field approach used, we cannot observe this full conversion, because the system is stuck in the coherent superposition state. Still, because of the many parallels observed between this coherent charge-transfer process on one side and adiabatic pulses and chirped laser pulse experiments on the other side, our results support a nonadiabatic conversion via adiabatic passage (NCAP) process.

Exchange of Angular Momentum. Because the system evolves from one quantum state to another, a change of quantum number is involved. Normally, transitions between electronic states follow selection rules, leading to transitions being allowed only via, for example, the release or absorption of a photon carrying an angular momentum. Also for the nonradiative exciton to charge-transfer transition in our study the change in quantum number has to be accompanied by a change of an associated physical quantity. In a publication by 
Purchase et al., the authors stressed the importance of convergence of time scales of nuclear and electronic circulating motion in a molecule for coherent charge transfer and suggested the exchange of angular momentum between the electronic and nuclear subsystems in a semiclassical coherent process. $^{32}$ Coupling of quantum and classical rotation is wellestablished in other fields of chemistry and chemical physics, where it has been shown that transitions in quantum subspaces give rise to observable rotations in a suitable interaction frame of the classical motion. ${ }^{43}$ Because in the Ehrenfest simulations, the conservation of total angular momentum is not enforced, we rather monitor collective motion in both the electronic as well as nuclear subsystems by following the orientational change in the electronic and nuclear components of the dipole moment, which we introduce here as a descriptive parameter for the process. The generated charge-transfer state results in a large electronic dipole moment change along the $x$-axis, potentially masking any other orientational change. Therefore, we focus on the dipole moment associated with the $\alpha$-electrons because they are not directly involved in the charge-transfer process and yet still respond to the electron transfer. The orientation of this dipole moment in the $x-y$ plane during the Ehrenfest dynamics with symmetric starting conditions is shown in Figure 3a. Within the first $\sim 35 \mathrm{fs}$, the dipole moment changes only along the $x$-axis, conserving symmetry; upon the breaking of symmetry the dipole moment starts to deviate from its initial orientation along the $x$-axis. At around $45 \mathrm{fs}$, when the electron-transfer process starts, a clear clockwise rotation with an angular momentum in the negative $z$-axis direction starts.

According to Purchase et al., this change in electronic angular momentum should be associated with a net rotation in the interaction frame of the nuclear motion of the coupled mode. ${ }^{32}$ We should therefore see the same rotational behavior if we plot the dipole moment orientation change of all the nuclei involved in $\mathrm{N}-\mathrm{H}$ covalent bond vibrations, which we have shown to be crucial for the coherent photoinduced charge transfer. The change in this nuclear dipole moment orientation reflects the net rotation in the interaction frame associated with the nuclear mode and is shown in Figure $3 \mathrm{~b}$. There is a clear correlation in the rotation of this nuclear dipole moment and the rotation of the dipole moment associated with the $\alpha$ electrons. After about 35 fs, the symmetry breaks, leading to deviation of both dipole moment orientations from the $x$-axis. At around $45 \mathrm{fs}$, on the onset of electron transfer, both the nuclear as well as electronic dipole moment start to rotate in unison in the molecular plane, maintaining correlation until about 80 fs into the simulation, where noise in the electronic system appears to obscure this connection. The change in quantum number therefore involves the gradual exchange of angular momentum between the electronic and nuclear system. For completeness, the dipole moment associated with the $\beta$ spin density in the symmetric case and the dipole moment associated with the $\alpha$-electrons in the non-symmetric simulation are shown in Figures S10 and S11.

In summary, the photoinduced coherent charge transfer in this donor-acceptor system can be described as follows: Upon excitation onto the Franck-Condon region in the excited state localized on the donor molecule, the system relaxes toward lower energetic regions of the excited state PES. During this relaxation, the system explores regions of strong coupling between the excitonic and lower-energy charge-transfer states as they approach each other energetically. As soon as the
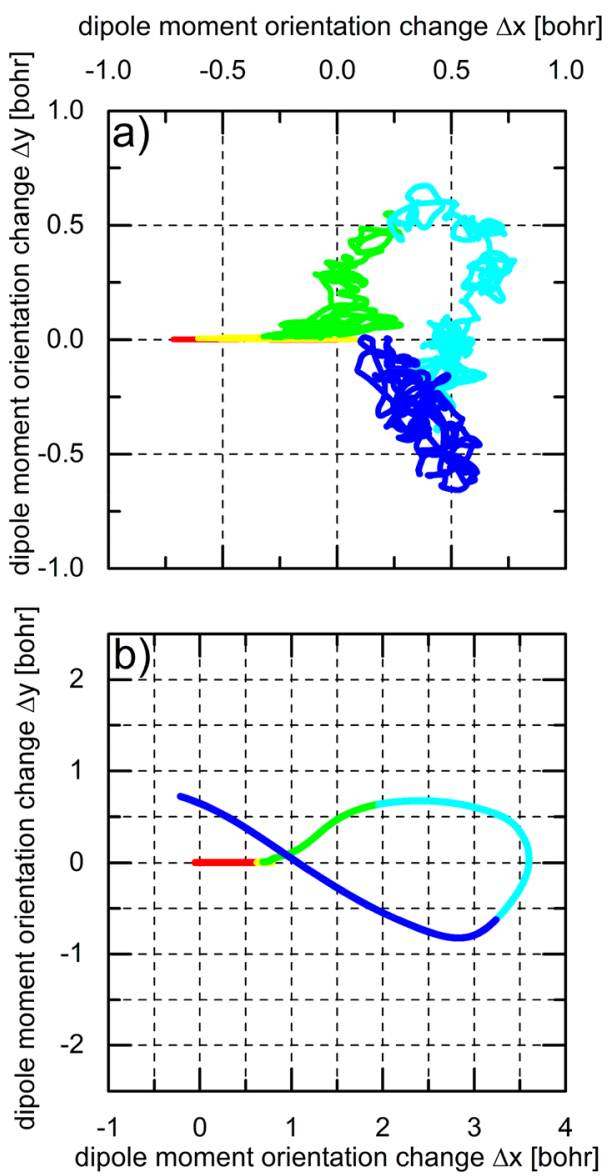

Figure 3. (a) Orientation of the dipole moment associated with $\alpha$ electrons in the $x-y$ plane for the simulation starting with enforced symmetric geometry. The colors correspond to consecutive time intervals, starting from red $(0-20 \mathrm{fs})$ to yellow $(20-40 \mathrm{fs})$, green (40-60 fs), light blue (60-80 fs), and dark blue (80-100 fs). The rotational character starting around $40 \mathrm{fs}$ is clearly visible. (b) Change of orientation of the dipole moment associated with the nitrogen and hydrogen nuclei involved in the $\mathrm{N}-\mathrm{H}$ stretches coupled to the electron-transfer process. The colors correspond to consecutive time intervals, starting from red $(0-20 \mathrm{fs})$ to yellow $(20-40 \mathrm{fs})$, green (40-60 fs), light blue (60-80 fs), and dark blue (80-100 fs). The correlation between this nuclear dipole moment orientation and the electronic dipole moment orientation in panel a is apparent.

energy difference between two states (one occupied, one unoccupied) is in resonance with a nuclear vibration available in the system, the nuclear vibration couples to the electronic motion and drives the electron transfer. A crucial condition for this process is the dynamic breaking of symmetry. Furthermore, the change in quantum number associated with the nonradiative transition between quantum states leads to a net exchange of angular momentum into the coupled vibrational state. Which particular nuclear mode first reaches the resonance condition depends on the specific potential energy surfaces involved in the electron-transfer process. Thus, the process is robust as it self-selects a rapid channel to the output. In the melamine-isocyanuric acid system the highest $\mathrm{N}-\mathrm{H}$ bond frequencies are the first and most important modes reaching the resonance condition. Because of the energetic gap between the $\mathrm{N}-\mathrm{H}$ stretches and all other modes, the $\mathrm{N}-\mathrm{H}$ bonds drive the electron transfer almost exclusively. This results in a relatively clean oscillatory pattern in the electrontransfer process. When exchanging hydrogens by deuterium, 
the system relaxes to a region where the involved states are closer in energy. The difference between the N-D stretches and the lower-frequency modes is now smaller, and thus, additional modes can drive the charge transfer. Therefore, more frequencies are involved and a more complex electrontransfer pattern emerges. In spite of the specificity of this donor-acceptor complex, we believe these principles are playing a role in natural systems and might help interpreting experimental data on coherent charge transfer.

\section{ASSOCIATED CONTENT}

\section{S Supporting Information}

The Supporting Information is available free of charge on the ACS Publications website at DOI: 10.1021/acs.jpclett.9b02408.

Computational details, TDDFT excitation energies, Ehrenfest dynamics with frozen nuclear geometry, assignment of relevant peaks in the VDOS, and more details on dynamic symmetry breaking and exchange of angular momentum (PDF)

Movie S1: Charge separation in the melamineisocyanuric acid pseudo base pair; shown in blue excess of $\beta$-spin density, representing the photoexcited electron; in red, a lack of $\beta$-spin density representing the hole (AVI)

Movie S2: Change of dipole moment orientation for the specified electronic and nuclear components in the $x-y$ plane over time together with the electron transfer (AVI)

\section{AUTHOR INFORMATION Corresponding Author \\ *E-mail: f.buda@chem.leidenuniv.nl. ORCID}

Huub J. M. de Groot: 0000-0002-8796-1212

Francesco Buda: 0000-0002-7157-7654

Notes

The authors declare no competing financial interest.

\section{ACKNOWLEDGMENTS}

This research has been financially supported by the NWO Solar to Products program (Project Number 733.000.007). We acknowledge the use of supercomputer facilities at SURFsara sponsored by NWO Physical Sciences, with financial support from The Netherlands Organization for Scientific Research (NWO).

\section{REFERENCES}

(1) Groot, M. L.; Pawlowicz, N. P.; van Wilderen, L. J. G. W.; Breton, J.; van Stokkum, I. H. M.; van Grondelle, R. Initial Electron Donor and Acceptor in Isolated Photosystem II Reaction Centers Identified with Femtosecond Mid-IR Spectroscopy. Proc. Natl. Acad. Sci. U. S. A. 2005, 102, 13087-13092.

(2) Durrant, J. R.; Klug, D. R.; Kwa, S. L.; van Grondelle, R.; Porter, G.; Dekker, J. P. A Multimer Model for P680, the Primary Electron Donor of Photosystem II. Proc. Natl. Acad. Sci. U. S. A. 1995, 92, 4798-4802.

(3) Romero, E.; van Stokkum, I. H. M.; Novoderezhkin, V. I.; Dekker, J. P.; van Grondelle, R. Two Different Charge Separation Pathways in Photosystem II. Biochemistry 2010, 49, 4300-4307.

(4) Faunce, T. A.; Lubitz, W.; Rutherford, A. W.; Bill; MacFarlane, D.; Moore, G. F.; Yang, P.; Nocera, D. G.; Moore, T. A.; Gregory, D. H.; Fukuzumi, S.; et al. Energy and Environment Policy Case for a
Global Project on Artificial Photosynthesis. Energy Environ. Sci. 2013, 6, 695-698.

(5) Faunce, T.; Styring, S.; Wasielewski, M. R.; Brudvig, G. W.; Rutherford, A. W.; Messinger, J.; Lee, A. F.; Hill, C. L.; deGroot, H.; Fontecave, M.; et al. Artificial Photosynthesis as a Frontier Technology for Energy Sustainability. Energy Environ. Sci. 2013, 6, 1074-1076.

(6) Tachibana, Y.; Vayssieres, L.; Durrant, J. R. Artificial Photosynthesis for Solar Water-Splitting. Nat. Photonics 2012, 6, 511-518.

(7) Kim, D.; Sakimoto, K. K.; Hong, D.; Yang, P. Artificial Photosynthesis for Sustainable Fuel and Chemical Production. Angew. Chem., Int. Ed. 2015, 54, 3259-3266.

(8) Yu, Z.; Li, F.; Sun, L. Recent Advances in Dye-Sensitized Photoelectrochemical Cells for Solar Hydrogen Production Based on Molecular Components. Energy Environ. Sci. 2015, 8, 760-775.

(9) Scholes, G. D. Coherence from Light Harvesting to Chemistry. J. Phys. Chem. Lett. 2018, 9, 1568-1572.

(10) Jumper, C. C.; Rafiq, S.; Wang, S.; Scholes, G. D. From Coherent to Vibronic Light Harvesting in Photosynthesis. Curr. Opin. Chem. Biol. 2018, 47, 39-46.

(11) Scholes, G. D.; Fleming, G. R.; Olaya-Castro, A.; van Grondelle, R. Lessons from Nature about Solar Light Harvesting. Nat. Chem. 2011, 3, 763-774.

(12) Romero, E.; Augulis, R.; Novoderezhkin, V. I.; Ferretti, M.; Thieme, J.; Zigmantas, D.; van Grondelle, R. Quantum Coherence in Photosynthesis for Efficient Solar-Energy Conversion. Nat. Phys. 2014, 10 (9), 676-682.

(13) Scholes, G. D.; Fleming, G. R.; Chen, L. X.; Aspuru-Guzik, A.; Buchleitner, A.; Coker, D. F.; Engel, G. S.; van Grondelle, R.; Ishizaki, A.; Jonas, D. M.; et al. Using Coherence to Enhance Function in Chemical and Biophysical Systems. Nature 2017, 543, 647-656.

(14) Chenu, A.; Scholes, G. D. Coherence in Energy Transfer and Photosynthesis. Annu. Rev. Phys. Chem. 2015, 66, 69-96.

(15) Engel, G. S.; Calhoun, T. R.; Read, E. L.; Ahn, T.-K.; Mančal, T.; Cheng, Y.-C.; Blankenship, R. E.; Fleming, G. R. Evidence for Wavelike Energy Transfer through Quantum Coherence in Photosynthetic Systems. Nature 2007, 446, 782-786.

(16) Fuller, F. D.; Pan, J.; Gelzinis, A.; Butkus, V.; Senlik, S. S.; Wilcox, D. E.; Yocum, C. F.; Valkunas, L.; Abramavicius, D.; Ogilvie, J. P. Vibronic Coherence in Oxygenic Photosynthesis. Nat. Chem. 2014, 6, 706-711.

(17) Collini, E.; Wong, C. Y.; Wilk, K. E.; Curmi, P. M. G.; Brumer, P.; Scholes, G. D. Coherently Wired Light-Harvesting in Photosynthetic Marine Algae at Ambient Temperature. Nature 2010, 463, 644-647.

(18) Tiwari, V.; Peters, W. K.; Jonas, D. M. Electronic Resonance with Anticorrelated Pigment Vibrations Drives Photosynthetic Energy Transfer Outside the Adiabatic Framework. Proc. Natl. Acad. Sci. U. S. A. 2013, 110, 1203-1208.

(19) Thyrhaug, E.; Tempelaar, R.; Alcocer, M. J. P.; Žídek, K.; Bína, D.; Knoester, J.; Jansen, T. L. C.; Zigmantas, D. Identification and Characterization of Diverse Coherences in the Fenna-MatthewsOlson Complex. Nat. Chem. 2018, 10, 780-786.

(20) Ma, F.; Romero, E.; Jones, M. R.; Novoderezhkin, V. I.; van Grondelle, R. Vibronic Coherence in the Charge Separation Process of the Rhodobacter Sphaeroides Reaction Center. J. Phys. Chem. Lett. 2018, 9, 1827-1832.

(21) Chin, A. W.; Prior, J.; Rosenbach, R.; Caycedo-Soler, F.; Huelga, S. F.; Plenio, M. B. The Role of Non-Equilibrium Vibrational Structures in Electronic Coherence and Recoherence in PigmentProtein Complexes. Nat. Phys. 2013, 9, 113-118.

(22) Eisenmayer, T. J.; Buda, F. Real-Time Simulations of Photoinduced Coherent Charge Transfer and Proton-Coupled Electron Transfer. ChemPhysChem 2014, 15, 3258-3263.

(23) Akimov, A. V.; Neukirch, A. J.; Prezhdo, O. V. Theoretical Insights into Photoinduced Charge Transfer and Catalysis at Oxide Interfaces. Chem. Rev. 2013, 113, 4496-4565.

(24) Monti, A.; Negre, C. F. A.; Batista, V. S.; Rego, L. G. C.; de Groot, H. J. M.; Buda, F. Crucial Role of Nuclear Dynamics for 
Electron Injection in a Dye-Semiconductor Complex. J. Phys. Chem. Lett. 2015, 6, 2393-2398.

(25) Falke, S. M.; Rozzi, C. A.; Brida, D.; Maiuri, M.; Amato, M.; Sommer, E.; De Sio, A.; Rubio, A.; Cerullo, G.; Molinari, E.; et al. Coherent Ultrafast Charge Transfer in an Organic Photovoltaic Blend. Science 2014, 344, 1001-1005.

(26) Andrea Rozzi, C.; Maria Falke, S.; Spallanzani, N.; Rubio, A.; Molinari, E.; Brida, D.; Maiuri, M.; Cerullo, G.; Schramm, H.; Christoffers, J.; et al. Quantum Coherence Controls the Charge Separation in a Prototypical Artificial Light-Harvesting System. Nat. Commun. 2013, 4, 1602.

(27) Park, M.; Im, D.; Rhee, Y. H.; Joo, T. Coherent and Homogeneous Intramolecular Charge-Transfer Dynamics of 1-TertButyl-6-Cyano-1,2,3,4-Tetrahydroquinoline (NTC6), a Rigid Analogue of DMABN. J. Phys. Chem. A 2014, 118, 5125-5134.

(28) Perdigão, L. M. A.; Champness, N. R.; Beton, P. H. Surface Self-Assembly of the Cyanuric Acid-Melamine Hydrogen Bonded Network. Chem. Commun. 2006, 0, 538-540.

(29) Prokhorenko, V. I.; Picchiotti, A.; Pola, M.; Dijkstra, A. G.; Miller, R. J. D. New Insights into the Photophysics of DNA Nucleobases. J. Phys. Chem. Lett. 2016, 7 (22), 4445-4450.

(30) Tannús, G.; Garwood, M. Adiabatic Pulses. NMR Biomed. 1997, 10, 423.

(31) Wollenhaupt, M.; Präkelt, A.; Sarpe-Tudoran, C.; Liese, D.; Baumert, T. Quantum Control by Selective Population of Dressed States Using Intense Chirped Femtosecond Laser Pulses. Appl. Phys. B: Lasers Opt. 2006, 82, 183-188.

(32) Purchase, R. L.; de Groot, H. J. M. Biosolar Cells: Global Artificial Photosynthesis Needs Responsive Matrices with Quantum Coherent Kinetic Control for High Yield. Interface Focus 2015, 5, 20150014.

(33) Purchase, R.; Cogdell, R.; Breitling, F.; Stadler, V.; van Hulst, N.; Kramer, G.-J.; Ramirez, A.; Zwijnenberg, R.; Kallergi, A.; de Baan, J. B.; et al. Semi-Synthetic Responsive Matrices for Artificial Photosynthesis. In Bioinspired Chemistry; Series on Chemistry, Energy and the Environment; World Scientific: Singapore, 2019; pp 47-69.

(34) Becke, A. D. Density-Functional Exchange-Energy Approximation with Correct Asymptotic Behavior. Phys. Rev. A: At., Mol., Opt. Phys. 1988, 38, 3098-3100.

(35) Lee, C.; Yang, W.; Parr, R. G. Development of the ColleSalvetti Correlation-Energy Formula into a Functional of the Electron Density. Phys. Rev. B: Condens. Matter Mater. Phys. 1988, 37, 785789.

(36) Andrade, X.; Alberdi-Rodriguez, J.; Strubbe, D. A.; Oliveira, M. J. T.; Nogueira, F.; Castro, A.; Muguerza, J.; Arruabarrena, A.; Louie, S. G.; Aspuru-Guzik, A.; et al. Time-Dependent Density-Functional Theory in Massively Parallel Computer Architectures: The Octopus Project. J. Phys.: Condens. Matter 2012, 24, 233202.

(37) Castro, A.; Appel, H.; Oliveira, M.; Rozzi, C. A.; Andrade, X.; Lorenzen, F.; Marques, M. a. L.; Gross, E. K. U.; Rubio, A. Octopus: A Tool for the Application of Time-Dependent Density Functional Theory. Phys. Status Solidi B 2006, 243, 2465-2488.

(38) Marques, M. A. L.; Castro, A.; Bertsch, G. F.; Rubio, A. Octopus: A First-Principles Tool for Excited Electron-Ion Dynamics. Comput. Phys. Commun. 2003, 151, 60-78.

(39) Castro, A.; Marques, M. A. L.; Rubio, A. Propagators for the Time-Dependent Kohn-Sham Equations. J. Chem. Phys. 2004, 121, 3425-3433.

(40) Schnedermann, C.; Yang, X.; Liebel, M.; Spillane, K. M.; Lugtenburg, J.; Fernández, I.; Valentini, A.; Schapiro, I.; Olivucci, M.; Kukura, P.; et al. Evidence for a Vibrational Phase-Dependent Isotope Effect on the Photochemistry of Vision. Nat. Chem. 2018, 10, 449455 .

(41) Eisenmayer, T. J.; de Groot, H. J. M.; van de Wetering, E.; Neugebauer, J.; Buda, F. Mechanism and Reaction Coordinate of Directional Charge Separation in Bacterial Reaction Centers. J. Phys. Chem. Lett. 2012, 3, 694-697.

(42) Moore, L. J.; Zhou, H.; Boxer, S. G. Excited-State Electronic Asymmetry of the Special Pair in Photosynthetic Reaction Center
Mutants: Absorption and Stark Spectroscopy. Biochemistry 1999, 38, 11949-11960.

(43) Boender, G. J.; Vega, S.; de Groot, H. J. M. A Physical Interpretation of the Floquet Description of Magic Angle Spinning Nuclear Magnetic Resonance Spectroscopy. Mol. Phys. 1998, 95, 921-934. 